\title{
Development and Implementation of the AIDA International Registry for Patients with Non- Infectious Uveitis
}

Francesca Della Casa $\cdot$ Antonio Vitale $\cdot$ Silvana Guerriero $\cdot$ Jurgen Sota $\cdot$ Rolando Cimaz

Gaafar Ragab · Piero Ruscitti · Rosa Maria R. Pereira · Francesca Minoia • Emanuela Del Giudice ·

Giacomo Emmi · Claudia Lomater · Sara Monti · Claudia Canofari · Carla Gaggiano ·

Giovanni Alessio $\cdot$ Elisabetta Miserocchi $\cdot$ Alessandro Conforti $\cdot$ Marilia A. Dagostin ·

Chiara Mapelli · Maria Pia Paroli · Veronica Parretti · Valeria Albano · Rosa Favale ·

Luca Marelli · Mohamed Tharwat Hegazy · Paola Cipriani · Isabele P. B. Antonelli ·

Valeria Caggiano $\cdot$ Emma Aragona $\cdot$ Ahmed Hatem Laymouna $\cdot$ Gian Marco Tosi $\cdot$

Maria Tarsia • Marco Cattalini · Francesco La Torre · Giuseppe Lopalco · Ewa Więsik-Szewczyk ·

Micol Frassi · Stefano Gentileschi · Heitor F. Giordano · Bruno Frediani · Samuel K. Shinjo ·

Donato Rigante · Petros P. Sfikakis · Alberto Balistreri - Mohamed A. Hussein · Rana Hussein Amin ·

Luca Cantarini (D) C Claudia Fabiani ·

Received: November 23, 2021 / Accepted: January 10, 2022 / Published online: January 31, 2022

(c) The Author(s) 2022

\section{ABSTRACT}

Introduction: The aim of this paper is to point out the design, development and deployment of the AutoInflammatory Disease Alliance

Luca Cantarini and Claudia Fabiani contributed equally to this manuscript.

Manuscript from the AIDA (AutoInflammatory Diseases Alliance) International Network (https://aidanetwork. org/en/).

\section{F. D. Casa}

Section of Clinical Immunology, Department of Translational Medical Sciences, University of Naples Federico II, Naples, Italy

A. Vitale · J. Sota $\cdot$ C. Gaggiano · V. Parretti . V. Caggiano - M. Tarsia - L. Cantarini $(\bowtie)$ Rheumatology Unit, Policlinico "Le Scotte", Department of Medical Sciences, Surgery and Neurosciences, Research Center of Systemic Autoinflammatory Diseases and Behçet's Disease Clinic, University of Siena, viale Bracci 16, 53100 Siena, Italy

e-mail: cantariniluca@hotmail.com
(AIDA) International Registry for paediatric and adult patients with non-infectious uveitis (NIU).

Methods: This is a physician-driven, population- and electronic-based registry implemented for both retrospective and prospective collection of real-world demographics, clinical, laboratory, instrumental and socioeconomic data of patients with uveitis and other non-infectious inflammatory ocular diseases recruited through the AIDA Network. Data recruitment, based on

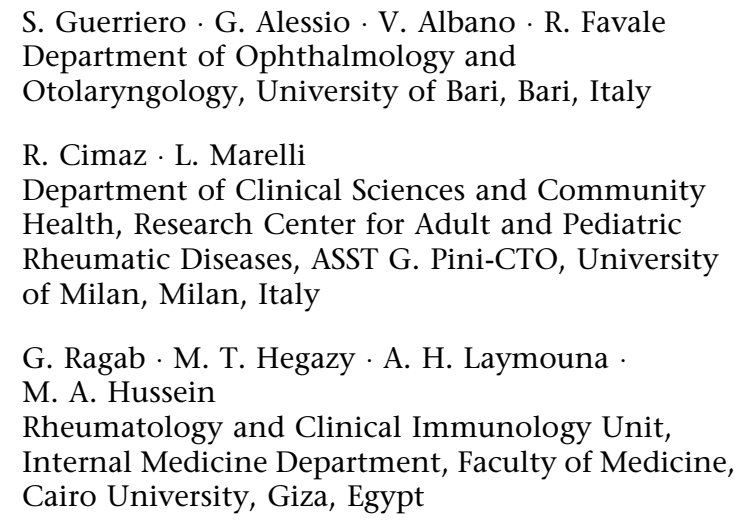


the Research Electronic Data Capture (REDCap) tool, is thought to collect standardised information for real-life research and has been developed to change over time according to future scientific acquisitions and potentially communicate with other similar instruments. Security, data quality and data governance are cornerstones of this platform.

Results: Ninety-five centres have been involved from 19 countries and four continents from 24 March to 16 November 2021. Forty-eight out of 95 have already obtained the approval from their local ethics committees. At present, the platform counts 259 users (95 principal investigators, 160 site investigators, 2 lead investigators, and 2 data managers). The AIDA Registry collects baseline and follow-up data using 3943 fields organised into 13 instruments, including

G. Ragab · M. T. Hegazy

Faculty of Medicine, Newgiza University (NGU), Cairo, Egypt

P. Ruscitti · A. Conforti · P. Cipriani Rheumatology Unit, Department of

Biotechnological \& Applied Clinical Sciences,

University of L'Aquila, L'Aquila, Italy

R. M. R. Pereira · M. A. Dagostin · I.

P. B. Antonelli · H. F. Giordano - S. K. Shinjo Rheumatology Division, Hospital das Clinicas (HCFMUSP), Faculdade de Medicina, Universidade de Sao Paulo, São Paulo, Brazil

F. Minoia

Pediatric Rheumatology, Fondazione IRCCS $\mathrm{Ca}^{\prime}$

Granda Ospedale Maggiore Policlinico, Milan, Italy

E. Del Giudice

Department of Maternal Infantile and Urological

Sciences, Sapienza University of Rome, Polo

Pontino, Rome, Italy

G. Emmi

Department of Experimental and Clinical Medicine, University of Florence, Florence, Italy

C. Lomater

UO Reumatologia, AO Ordine Mauriziano, Turin, Italy patient's demographics, history, symptoms, trigger/risk factors, therapies and healthcare utilization for patients with NIU.

Conclusions: The development of the AIDA Registry for patients with NIU will facilitate the collection of standardised data leading to realworld evidence and enabling international multicentre collaborative research through inclusion of patients and their families worldwide.

Keywords: Autoinflammatory diseases; Clinical management; Innovative biotechnologies; International registry; Personalised medicine; Precision medicine; Rare diseases; Uveitis

S. Monti

Rheumatology Department, IRCCS Policlinico S.

Matteo Foundation, Pavia, Italy

S. Monti

Experimental Medicine, University of Pavia, Pavia, Italy

C. Canofari

U.O.C. Reumatologia, Ospedale San Camillo-

Forlanini, Roma, Italy

E. Miserocchi

Department of Ophthalmology, San Raffaele Scientific Institute, IRCCS Ospedale San Raffaele, Vita-Salute San Raffaele University, Milan, Italy

C. Mapelli

Ophthalmological Unit, Fondazione IRCCS $\mathrm{Ca}^{\prime}$ Granda Ospedale Maggiore Policlinico di Milano, Milan, Italy

M. P. Paroli

Uveitis Unit, Department of Sense Organs, Eye Clinic, Sapienza University of Rome, Rome, Italy

E. Aragona

Division of Gastroenterology, Ospedali Riuniti Villa Sofia-Vincenzo Cervello, Palermo, Italy 


\section{Key Summary Points}

The AIDA International Registry for noninfectious uveitis and other ocular inflammatory conditions is currently available and ready for data collection.

The AIDA Registry will allow scientific communities to cooperate, share experience and actively participate in research projects.

Implementation and execution of this large international registry will overcome limitations due to the rarity of ocular inflammatory diseases.

Real-life data from the AIDA Registry will improve both clinical management and therapeutic approach of patients with non-infectious uveitis.

G. M. Tosi · C. Fabiani

Ophthalmology Unit, Department of Medicine, Surgery and Neurosciences, University of Siena, Siena, Italy

M. Cattalini

Pediatric Clinic, University of Brescia and Spedali Civili di Brescia, Brescia, Italy

F. La Torre

Clinical Pediatrics, University of Bari, Bari, Italy

G. Lopalco

Rheumatology Unit, Department of Emergency and Organ Transplantation, University of Bari, Bari, Italy

E. Więsik-Szewczyk

Department of Internal Medicine, Pulmonology, Allergy and Clinical Immunology, Central Clinical Hospital of the Ministry of National Defence, Military Institute of Medicine, Warsaw, Poland

\section{Frassi}

Rheumatology and Clinical Immunology, Spedali Civili and Department of Clinical and Experimental Sciences, University of Brescia, Brescia, Italy

S. Gentileschi · B. Frediani

Unit of Rheumatology, Azienda Ospedaliero-

Universitaria Senese, Siena, Italy

\section{INTRODUCTION}

Uveitis represents a group of challenging conditions that greatly impact patients' quality of life and potentially induce sight-threatening sequelae and even blindness. The term 'uveitis' refers to a broad spectrum of clinical entities characterized by the presence of uveal inflammation. Indeed, all the structures which make up the uveal tract (iris, ciliary body and choroid) can be affected, and besides, the inflammatory process can eventually extend to adjacent tissues, involving the sclera, vitreous, retina and optic nerve [1]. Non-infectious uveitis (NIU) can occur in all age groups and often affects young adults [2-4]. The spectrum of NIU deserves a detailed re-evaluation especially in light of the increasing knowledge in terms of diagnostic and therapeutic opportunities.

Rare diseases, such as NIU, usually present a reduced epidemiological burden that leads to poor availability of patients for recruitment into clinical trials, and therefore a lack of standardised care and knowledge. In this regard, the

\section{Rigante}

Department of Life Sciences and Global Health, Fondazione Policlinico Universitario A. Gemelli IRCCS, Rome, Italy

\section{Rigante}

Rare Diseases and Periodic Fevers Research Centre, Università Cattolica Sacro Cuore, Rome, Italy

\section{P. P. Sfikakis}

Joint Academic Rheumatology Program, 1st Department of Propedeutic Internal Medicine, School of Medicine, National and Kapodistrian University of Athens, Athens, Greece

\section{A. Balistreri}

Bioengineering and Biomedical Data Science Lab, Department of Medical Biotechnologies, University of Siena, Siena, Italy

\section{R. H. Amin}

Opthalmology Department, Faculty of Medicine, Cairo University, Giza, Egypt 
AutoInflammatory Disease Alliance (AIDA) network has been developed with the primary aim to gather a worldwide group of physicians and researchers interested in sharing knowledge, experience, information and different perceptions on the clinical, therapeutic and research approaches to rare diseases such as autoinflammatory disorders. The focus of the AIDA project is the development and maintenance of international registries for patients with ocular inflammatory diseases, but also with monogenic and multifactorial autoinflammatory disorders, as familial Mediterranean fever, Still's disease, Behçet's disease. To date, nine registries have been developed, each corresponding to a multicentre, non-interventional, observational cohort clinical study designed to investigate specific inflammatory diseases. The following registries are current available: (i) NIU, (ii) non-infectious scleritis, (iii) Behçet's disease, (iv) monogenic autoinflammatory diseases, (v) Still's disease, (vi) Schnitzler's syndrome, (vii) periodic fever, aphthous stomatitis, pharyngitis and cervical adenitis (PFAPA) syndrome, (viii) vacuoles/E1 enzyme/X-linked autoinflammatory, somatic (VEXAS) syndrome and (ix) undifferentiated systemic autoinflammatory diseases (USAIDs). The registries may be reached at the following webpage: https://aidanetwork.org/en/registries.

The main purpose of this paper is to point out the design, development and deployment of the AIDA International Registry specifically dedicated to patients with NIU and other ocular inflammatory disorders unrelated to Behçet's disease or scleritis, for which other specific AIDA registries are available. As NIU ranks among the leading causes of blindness in the middle-aged population of developed countries, accounting for about $15 \%$ of preventable loss of vision [5], this international registry represents a crucial source of data covering natural history, epidemiology and medical information capable of overcoming the limits of traditional data collection and the limitations related to the small number of patients available at each single reference centre.

\section{METHODS}

\section{Study Design}

Similar to other AIDA registries, the NIU one is a physician-driven, population- and electronicbased registry, built for paediatric and adult patients suffering from uveitis and other noninfectious inflammatory eye diseases.

Data are collected both retrospectively (information already available at the time of the enrolment into the AIDA Registry) and prospectively (information obtained after the inclusion into the AIDA Registry). Prospective data must be updated at least once a year or in the case of treatment change (including posology adjustments and combination of different immunosuppressant agents) and at 3-month, 6-month and 12-month follow-up assessments after treatment changing.

In accordance with its observational nature, the AIDA Registry captures demographic, genetic, clinical, laboratory, instrumental and treatment details. Long-term outcomes and prognostic variables are also collected as long as patients do not withdraw their consent to the project over time. Only data derived from the standard routine management are recorded, and no additional laboratory, clinical or instrumental investigations will be performed as a result of participation in the project. Any treatment approach administered prior or after the enrolment and each posology change performed for the patients' welfare should be described in the registry; however, none of the prescribed therapies must be determined by adherence to the study in any way.

The participation is open to any centre that manages patients with NIU. In order to participate it is mandatory to obtain the approval from the local ethics committee and appoint a principal investigator to coordinate the study locally and site investigators responsible for the documentation and data entry for that site. Since the gathered data refer to information routinely collected for the best standard of care, there is neither cost nor financial compensation to participate. After having obtained the local ethics committee approval and provided other 
requirements, the centres may obtain credentials to access the registry and share information.

\section{Registry Objectives}

Primary aim of this registry is to overcome the fragmentation of clinical and research experience of inflammatory ocular diseases in order to carry out solid research based on a large number of patients and obtain reliable results with pioneering studies about the optimal clinical and therapeutic management of these patients distinguished according to the specific diagnosis, the background clinical setting, and specific demographic clinical and instrumental features. Further aims include (I) evaluation of new associations between non-infectious ocular inflammation and systemic immune-mediated disorders; (II) the response to different locoregional, intravitreal and systemic therapies looking for differences based on demographic, clinical and instrumental features; (III) the description of the socioeconomic impact of the disease in relation to both the epidemiologic burden in different geographic contexts and benefits obtained with treatment approaches currently available; (IV) the behaviour of NIU during pregnancy and the post-partum period; (V) the search for any variable associated with severity of the systemic and/or ocular disease in order to identify patients needing a more careful follow-up assessment early; (VI) the identification of variables capable of the early identification of patients more likely to be responsive to different treatments approaches; (VII) the behaviour and impact on eyesight of inflammatory-related ocular complications in the light of the new diagnostic and therapeutic opportunities; (VIII) the identification of predisposing factors for NIU, severity of manifestations and response to treatments; (IX) the assessment of current clinimetric tools created to evaluate patients with intraocular inflammation; (X) evaluation of the use of currently available clinimetric tools in different diseases and contexts along with the search for new clinimetric instruments aimed at the current and prognostic assessment of ocular involvement. Noteworthy, data collection will clarify the worldwide distribution of patients, thus facilitating the recruitment of patients in future randomized controlled trials (RCTs).

All the objectives of the registry will be considered in the light of the machine learning data analysis, which represents a new way to evaluate clinical information and real-world data by automating data collection and assessing healthcare big data. This approach will pave the way for the optimisation of research and implementation of precision medicine. Table 1 summarize all objectives of the NIU registry.

\section{Inclusion/Exclusion Criteria}

Inclusion criteria into the AIDA Registry for NIU consist in the diagnosis of uveitis, strictly made by ophthalmologists trained in uveitis, and in the certain exclusion of infectious etiology (e.g. syphilis, tuberculosis, herpesvirus infection, toxoplasmosis, Lyme disease). Additionally, all patients enrolled have to provide their written informed consent after having been carefully informed about the project, its aims, the privacy related to security according to local and/or country regulations, and the lack of any impact of the study on clinical practice. The patient has to be informed that refusing to enter the study, or choosing to withdraw from it at any time, will not affect the clinical workflow at the reference centre. The parents, or a legally acceptable representative, and the adolescent subjects have to be willing and able to comply with the project requirements for the duration of the study.

The exclusions criteria are (I) the lack of informed consent/assent from the patient; (II) the identification of any infectious etiology explaining intraocular inflammation.

Of note, the AIDA project includes a specific registry for patients with scleritis and a registry dedicated to patients diagnosed with Behçet's disease: these registries gather specific items for such disorders, and patients with scleritis and Behçet's disease-related ocular inflammation should be included in these dedicated registries. 
Table 1 Summary of all the objectives of the registry dedicated to patients with non-infectious uveitis (NIU)

Main $\begin{gathered}\text { Built an international registry to overcome } \\ \text { objective }\end{gathered}$
limitation of small number of patients
with NIU in each single centre
objectives $\quad \begin{aligned} & \text { Analyse new associations between NIU and } \\ & \text { systemic immune-mediated disorders }\end{aligned}$
Study response to different locoregional,
intravitreal and systemic therapies
Evaluate socioeconomic impact of the
disease in different geographic contexts
Study NIU outcome during pregnancy and
post-partum
Analyse variables associated with severity of
the ocular disease
Analyse variables to allow early
identification of patients more responsive
to certain therapies
Study behaviour and impact on eyesight of
inflammatory-related ocular
complications in the light of the new
diagnostic and therapeutic opportunities
Search for NIU predisposing factors
Assessment of current clinimetric tools
aimed at the current and prognostic
assessment of ocular involvement

\section{Online Data Collection}

Data are collected through Research Electronic Data Capture (REDCap), an electronic data capture tool developed at the Vanderbilt University Medical Center (VUMC) and hosted at Virginia Commonwealth University (Award Number UL1TR002649), which can also be used to develop patient registries. The software is distributed at no cost to members of the REDCap Consortium, a global collaboration of over
5300 diverse institutions across 143 countries that have installed the software and collaborate to provide support [6].

Investigators included in the AIDA project are able to $\log$ in the registry through the REDCap web interface, insert data on the pages (instruments) of the registry and then review (and eventually complete) their own information. Neither principal investigators nor site investigators are allowed to see information inserted by other centres.

While the public website (https:// aidanetwork.org/en/) may be accessed by everyone who wants to learn about AIDA Network's relevance and how to participate in the project, the registry website (https://sitbio.med. unisi.it/redcap/redcap_v11.4.3/index.php?pid= 37 ) is hosted separately from the public website as a security measure. Data entry is passwordprotected and the collected data are stored on a secure server of the University of Siena, Siena, Italy. The electronic data entry system of the registry is in English.

\section{Ethics}

The first national regulatory approval of the AIDA project was obtained in June 2019 by the Ethics Committee of Azienda Ospedaliera Universitaria Senese, Siena, Italy (Ref. N. 14951). Later, different centres from Europe, the Middle East, North Africa and America approved the project with the aim to share information and research projects dealing with non-infectious ocular inflammation around the world.

The registry is run in compliance with the principles of the Declaration of Helsinki. Participation into the registry is voluntary. After having received age-appropriate information sheets, patients listed (or their parents/caregivers) have to give their informed consent; minors aged 12 years or older are also required to provide their assent before inclusion in the study. Both patients and principal investigators may withdraw their consent for the use of data for statistical analyses at any time. In case a patient withdraws the consent, no further data for that patient will be entered into the registry 


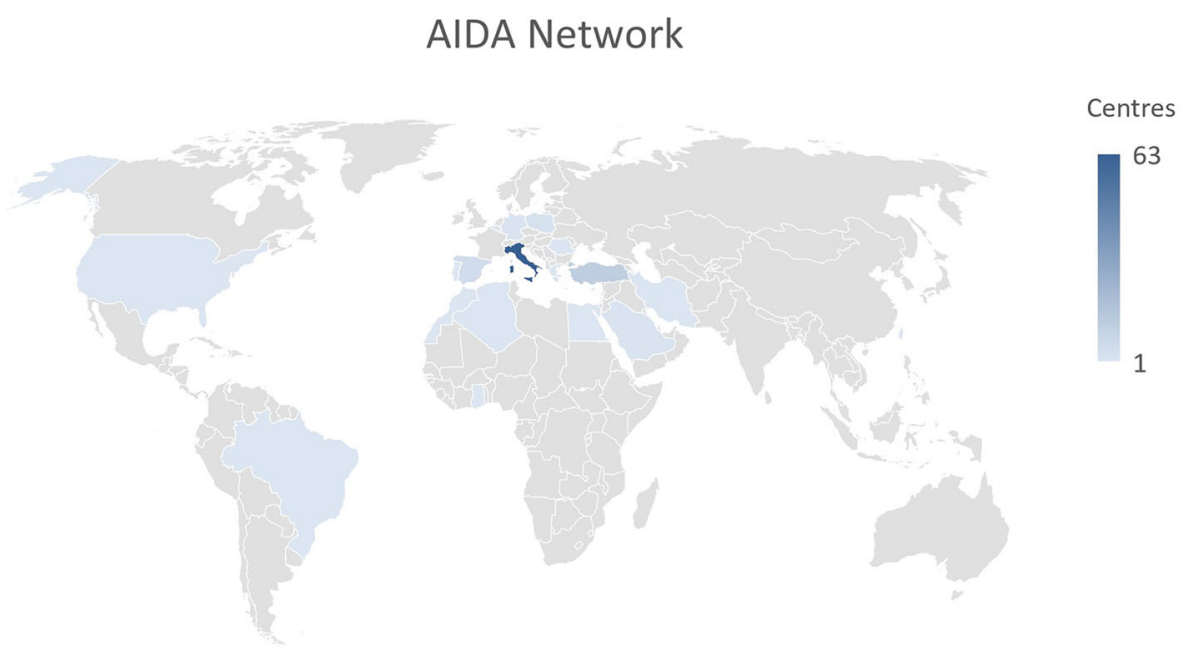

Fig. 1 Countries involved in the AIDA Network based on the number of centres for each country

and, if requested by the patient, all their prior data will be deleted from the registry soon after communication with the study promoter. Patients' data are kept in accordance with the EU General Data Protection Regulations (GDPR, $2016 / 679 / \mathrm{EU})$ or its counterpart worldwide on the processing of personal data and the protection of privacy in electronic communication [7].

Patients do not receive any honoraria or other types of payment for participation in the registry, and no links to billing of the healthcare system or insurance companies have to be highlighted.

\section{RESULTS}

Since its launch on 24 March 2021, the project has quickly reached a broad global reach, spreading to 19 countries (Algeria, Belgium, Brazil, Egypt, Germany, Ghana, Greece, Iran, Italy, Lebanon, Morocco, Poland, Portugal, Romania, Saudi Arabia, Spain, Taiwan, Turkey, the USA) and four continents. Ninety-five centres around the world have joined the AIDA project and 48 have already obtained the approval from the local ethics committee; as of 16 November 2021, 12/48 (25\%) have entered data on the registry dedicated to patients with NIU; 259 users (95 principal investigators, 160 site investigators, 2 lead investigators, 2 data managers).

The Countries worldwide involved in the AIDA Network are illustrated in Fig. 1. Centres involved in the AIDA Network are listed in detail at the following web page: https:// aidanetwork.org/en/clinical-sites.

\section{Registry Development}

Clinical variables included in the study have been chosen in order to reconstruct the regular course of a patient's complete clinical history along with their diagnostic, follow-up and therapeutic course; additional items have been included with the aim to solve the current unmet needs. In total, 3943 common data elements (fields) organised into 13 instruments (forms) currently constitute the registry.

The common data elements correspond to an equal number of items that describe data on the patient's demographics, medical history, laboratory features, association with other diseases, ocular involvement and associated systemic involvement, symptoms developing over time, comorbidities, cardiovascular risk, laboratory work-up, instrumental and ophthalmologic exams, pregnancies after the onset of uveitis or other inflammatory ocular manifestations, past and current treatments, long-term clinical outcomes, short- and long-term 
response to treatments, response to different treatment strategies in terms of posology changes and drug combinations, and access to healthcare. The fields are organized in such a way as to appear only if patient's clinical history makes it necessary to answer, owing to a branching mechanism of questions. Only a relatively small part of the 3943 fields will appear to the investigators for each patient, in relation to the patient's history.

Thanks to the REDCap tool's adaptability, the registry may be constantly improved in accordance with the new acquisitions derived from international medical literature in the future. The number of variables included in the registry will change in the future in accordance with the need to answer future scientific questions and to perform cutting-edge research.

Data elements, where appropriate, are shared with other AIDA registries; other bespoke data elements are specific to NIU and have been added to meet the specific field of this disease in terms of clinical manifestations, disease activity, possible complications, specific treatments, and different follow-up approach planned for the prospective enrolment phase. In relation to this last point, longitudinal data are captured through a specific follow-up instrument inclusive of the ophthalmologic assessment, evaluation of the systemic disease course (in nonidiopathic cases) and treatments performed in the previous time of observation. This registry has been designed so that it may communicate with other existing and future registries with similar purposes. Merging data among different registries will allow to answer current and future unmet needs and to assess inflammatory ocular diseases from different perspectives.

Both the retrospective and the prospective phases require the compilation of specific data drawn from the ophthalmic examinations, such as the Lens Opacities Classification System III (LOCSIII), best corrected visual acuity (BCVA), the classifications provided by the Standardization of Uveitis Nomenclature (SUN) Working Group, the grading scheme for vitreous haze according with Nussenblatt et al., the National Eye Institute Grading System for Vitreous Cells (adopted by SUN Working Group), the ASUWOG fluorescein angiography scoring system, and the ASUWOG indocyanine green angiographic scoring system [8-14].

Table 2 provides a full list of instruments constituting the registry and the time points to which they should refer.

\section{Patients' Enrolment into AIDA Registry}

The enrolment of patients with NIU on the corresponding AIDA Registry started on 24 March 2021. As of 16 November 2021, 309 patients (188 female/119 male; 2 missing values) have been enlisted.

\section{DISCUSSION}

The increasing use of electronic and informatic bioengineering in clinical research has allowed the deployment of online sources to quickly get essential data for scientific purposes, especially in the field of rare diseases characterized by a reduced epidemiological burden and huge difficulties with patient enrolment. In particular, case reports, case series and small retrospective studies about ocular inflammatory diseases were the only studies possible in the past decades, as the number of patients was relatively limited even in international reference centres. In recent times, the development of online platforms has enabled international centres around the world to share knowledge, information and patients' data. This has made it possible to carry out studies based on a sufficient number of patients and even RCTs, where the distribution of patients fulfilling inclusion criteria may be clear as soon as the study is designed. In this context, real-world data have recently emerged as a pivotal area of interest for researchers, as patient's behaviour, co-occurring treatments and environmental factors are not considered for recruitment in real-life clinical studies. This leads to more powerful insights regarding the application of results in routine clinical practice, as real-world settings are neither influenced nor limited by either protocol boundaries or enrolment of specific cohorts steered by conventional inclusion and exclusion criteria. In this scenario, the AIDA project has led to the implementation of the first online registry for 
Table 2 List of instruments (forms) included in the AIDA International Registry dedicated to patients with non-infectious uveitis, with the corresponding number of common data elements, time points to which they should refer and number of mandatory fields included

\begin{tabular}{|c|c|c|c|}
\hline Instruments & Fields & $\begin{array}{l}\text { Retrospective/ } \\
\text { prospective phase }\end{array}$ & $\begin{array}{l}\text { No. of } \\
\text { mandatory fields }\end{array}$ \\
\hline Demographics & 10 & Retrospective phase & 4 \\
\hline Consents & 4 & $\begin{array}{l}\text { Retrospective/ } \\
\text { prospective phase }\end{array}$ & 1 \\
\hline Diagnostic data and general information & 133 & Retrospective phase & 4 \\
\hline Features of uveitis and ocular history & 63 & Retrospective phase & 0 \\
\hline Cardiovascular risk & 18 & $\begin{array}{l}\text { Retrospective/ } \\
\text { prospective phase }\end{array}$ & 1 \\
\hline Data concerning fertility and pregnancy & 12 & $\begin{array}{l}\text { Retrospective/ } \\
\text { prospective phase }\end{array}$ & 1 \\
\hline Disease course and treatment during pregnancies & 60 & $\begin{array}{l}\text { Retrospective/ } \\
\text { prospective phase }\end{array}$ & 1 \\
\hline $\begin{array}{l}\text { Treatments performed during patient's history - the retrospective } \\
\text { phase }\end{array}$ & 69 & Retrospective phase & 0 \\
\hline Treatment with cDMARDs not associated with biologic agents & 393 & Retrospective phase & 6 \\
\hline $\begin{array}{l}\text { Treatment with small molecules not associated with biologic } \\
\text { agents - the retrospective phase }\end{array}$ & 654 & Retrospective phase & 12 \\
\hline Treatment with biotechnological agents-the retrospective phase & 986 & Retrospective phase & 17 \\
\hline $\begin{array}{l}\text { Ophthalmological assessment for each treatment performed-the } \\
\text { retrospective phase }\end{array}$ & 399 & Retrospective phase & 0 \\
\hline Follow-up visits-the prospective phase & 1118 & Prospective phase & 51 \\
\hline
\end{tabular}

cDMARD conventional disease-modifying antirheumatic drug

patients with NIU and other inflammatory ocular manifestations such as retinal vasculitis. As for other immune-mediated diseases, the management of NIU requires the collaboration of different specialties that must necessarily communicate with each other, including ophthalmologists, rheumatologists, geneticists, immunologists, gastroenterologists, dermatologists, internal medicine physicians, and paediatricians. For these reasons, this online tool reachable from all over the world can overcome the divisions due to distance as well as the fragmentation of knowledge and research in the field of ocular inflammation either idiopathic or related to immune-mediated disorders.
The AIDA project has already achieved the goal of developing an online platform dedicated to autoinflammatory diseases and inflammatory ocular diseases; this platform represents a virtual forum where the scientific community may easily get in touch, share experience, and actively participate in international research projects, without worrying about the number of patients to include or wasting time to specifically contact other centres. Therefore, the implementation and execution of a large international registry may finally overcome the limitations of smaller individual studies [15], providing valuable and generalizable results to apply in the routine management of patients. 
A large-scale, long-term, patient registry may provide additional evidence on the behaviour of NIU in the short and long term, according to geographical distribution, different treatments and features capable of identifying subgroups of patients who may behave differently in terms of prognosis and response to treatments. Furthermore, in a time when the need for personalised medicine aims at tailoring the daily care and treatments according to the specific features of the patient, future research has to aspire to identify clinical elements and variables useful for preventing the development of severe manifestations and complications. As a whole, personalised medicine will avoid both overmedication in patients with a lower risk for disease progression and overtreatment when a reduced probability of response to a given treatment may be identified early. Furthermore, it will be possible to characterize new associations between uveitis and any other systemic or genetic diseases, possibly reducing the number of patients diagnosed with idiopathic forms of uveitis.

All the objectives of the registry are even more pivotal in the light of the new diagnostic and treatment acquisitions. These aspects should be implemented by increasingly expanding the number of patients included in the registry, who should not be necessarily severe and complicated. The registry may also provide an invaluable source of data to better understand the behaviour of ocular inflammatory disorders during and after pregnancy, with specific attention on treatments to use in these periods and the specific trimester where ocular disease may be more severe. The prospective phase of the project will also provide more information on the socioeconomic impact of the disease and the benefits that national health systems can obtain from the treatments used. A further outcome of this registry concerns the application of currently used clinimetric tools and development of new instruments capable of assessing disease severity or the short- and longterm prognosis. The worldwide distribution of patients enrolled will allow the evaluation of the accuracy and specificity of these clinimetric tools according to the different geographical contexts and the patient's specific systemic disease as well as treatment used.

The AIDA Registry for patients with NIU has the usual limitations of observational research and registries. Of note, entering data into the registry requires time and attention, especially when the patient's medical history is complex, such as when they have undergone multiple treatment approaches and many posology changes, or when many relapses have to be described. Therefore, while entering prospective data from follow-up visits takes 10-15 min, entering retrospective data from a particularly complex case can take many hours. In addition, some ophthalmologic examinations required in the registry may be highly specialistic for nonophthalmologist investigators. In this regard, close cooperation with the referral ophthalmologist should be advocated. In addition, the participating investigators are under no obligation to consecutively register all their patients with NIU, and therefore unintended selection bias is possible. Nevertheless, beyond its limitations, this is the first international registry for patients with non-infectious inflammatory ocular diseases; the worldwide geographical basis guarantees the achievement of all objectives fixed.

In conclusion, the development of the AIDA International Registry for patients with NIU will facilitate sharing knowledge and information from the numerous centres involved with worldwide management of uveitis. The collection of standardised data will promote international multicentre collaborative research projects for both retrospective studies and RCT, as well as international consultation, and the possible active involvement of patients and their families. The use of real-life data obtained from this registry will allow evidence-based clinical management of patients with non-infectious inflammatory ocular diseases. The next step will include an implementation of patients' inclusion, a further dissemination of the network with inclusion of all possible stakeholders, and the start of studies from data already collected. 


\section{ACKNOWLEDGEMENTS}

Funding. No funding or sponsorship was received for this study or publication of this article.

Authorship. All named authors meet the International Committee of Medical Journal Editors (ICMJE) criteria for authorship for this article, take responsibility for the integrity of the work as a whole, and have given their approval for this version to be published.

Author Contributions. Francesca Della Casa wrote the first draft of the manuscript; Antonio Vitale conceived and designed the study and revised the manuscript; Claudia Fabiani conceived and designed the study and the non-infectious uveitis registry; Luca Cantarini conceived and designed the study and acts as AIDA Registries Coordinator; Silvana Guerriero, Jurgen Sota, Rolando Cimaz, Gaafar Ragab, Piero Ruscitti, Rosa Maria R. Pereira, Francesca Minoia, Emanuela Del Giudice, Giacomo Emmi, Claudia Lomater, Sara Monti, Claudia Canofari, Carla Gaggiano, Giovanni Alessio, Elisabetta Miserocchi, Alessandro Conforti, Marilia A. Dagostin, Chiara Mapelli, Maria Pia Paroli, Veronica Parretti, Valeria Albano, Rosa Favale, Luca Marelli, Mohamed Tharwat Hegazy, Paola Cipriani, Isabele P. B. Antonelli, Maria Tarsia and Rana Hussein Amin were involved in data recruitment in the registry dedicated to patients with uveitis and other inflammatory ocular diseases; Valeria Caggiano, Emma Aragona, Ahmed Hatem Laymouna, Gian Marco Tosi, Marco Cattalini, Francesco La Torre, Giuseppe Lopalco, Ewa Więsik-Szewczyk, Micol Frassi, Stefano Gentileschi, Heitor F. Giordano, Bruno Frediani, Samuel K. Shinjo, Petros P. Sfikakis, were included in the authorship as investigators from to the three top contributor centres for any of the other seven AIDA registries; Donato Rigante critically revised the draft of the paper; Alberto Balistreri is the bioengineer involved in the technical management of the platform and registries. Authorship has been established based on the number of data recruited in the AIDA registries on 16 November 2021.
Disclosures. Francesca Della Casa, Antonio Vitale, Silvana Guerriero, Jurgen Sota, Rolando Cimaz, Gaafar Ragab, Piero Ruscitti, Rosa Maria R. Pereira, Francesca Minoia, Emanuela Del Giudice, Giacomo Emmi, Claudia Lomater, Sara Monti, Claudia Canofari, Carla Gaggiano, Giovanni Alessio, Elisabetta Miserocchi, Alessandro Conforti, Marilia A. Dagostin, Chiara Mapelli, Maria Pia Paroli, Veronica Parretti, Valeria Albano, Rosa Favale, Luca Marelli, Mohamed Tharwat Hegazy, Paola Cipriani, Isabele P. B. Antonelli, Valeria Caggiano, Emma Aragona, Ahmed Hatem Laymouna, Gian Marco Tosi, Maria Tarsia, Marco Cattalini, Francesco La Torre, Giuseppe Lopalco, Ewa Więsik-Szewczyk, Micol Frassi, Stefano Gentileschi, Heitor F. Giordano, Bruno Frediani, Samuel K. Shinjo, Donato Rigante, Petros P. Sfikakis, Alberto Balistreri, Mohamed A. Hussein, Rana Hussein Amin, Luca Cantarini and Claudia Fabiani have nothing to disclose.

Compliance with Ethics Guidelines. The first national regulatory approval of the AIDA project was obtained in June 2019 by the Ethics Committee of Azienda Ospedaliera Universitaria Senese, Siena, Italy (Ref. N. 14951). Later, different centres from Europe, the Middle East, North Africa and America approved the project with the aim to share information and research projects dealing with non-infectious ocular inflammation around the world. The registry is run in compliance with the principles of the Declaration of Helsinki. Participation into the registry is voluntary. After having received ageappropriate information sheets, patients listed (or their parents/caregivers) have to give their informed consent; minors aged 12 years or older are also required to provide their assent before inclusion in the study. Both patients and principal investigators may withdraw their consent for the use of data for statistical analyses at any time. In case a patient withdraws the consent, no further data for that patient will be entered into the registry and, if requested by the patient, all their prior data will be deleted from the registry soon after communication with the study promoter. Patients' data are kept in accordance with the EU General Data Protection Regulations (GDPR, 2016/679/EU) or its 
counterpart worldwide on the processing of personal data and the protection of privacy in electronic communication [7]. Patients do not receive any honoraria or other types of payment for participation in the registry, and no links to billing of the healthcare system or insurance companies have to be highlighted.

Data Availability. The datasets generated during and/or analyzed during the current study are available from the corresponding author on reasonable request.

Open Access. This article is licensed under a Creative Commons Attribution-NonCommercial 4.0 International License, which permits any non-commercial use, sharing, adaptation, distribution and reproduction in any medium or format, as long as you give appropriate credit to the original author(s) and the source, provide a link to the Creative Commons licence, and indicate if changes were made. The images or other third party material in this article are included in the article's Creative Commons licence, unless indicated otherwise in a credit line to the material. If material is not included in the article's Creative Commons licence and your intended use is not permitted by statutory regulation or exceeds the permitted use, you will need to obtain permission directly from the copyright holder. To view a copy of this licence, visit http://creativecommons.org/licenses/by$\mathrm{nc} / 4.0 /$.

\section{REFERENCES}

1. Fabiani C, Vitale A, Orlando I, et al. Impact of uveitis on quality of life: a prospective study from a tertiary referral rheumatology-Ophthalmology Collaborative Uveitis Center in Italy. Isr Med Assoc J. 2017;19(8):478-83.

2. Rothova A, Buitenhuis HJ, Meenken C, et al. Uveitis and systemic disease. Br J Ophthalmol. 1992;76(3): 137-41. https://doi.org/10.1136/bjo.76.3.137.

3. Tsirouki T, Dastiridou A, Symeonidis C, et al. A focus on the epidemiology of uveitis. Ocul Immunol Inflamm. 2018;26(1):2-16. https://doi.org/10. 1080/09273948.2016.1196713.
4. Joltikov KA, Lobo-Chan AM. Epidemiology and risk factors in non-infectious uveitis: a systematic review. Front Med (Lausanne). 2021;10(8):695904. https://doi.org/10.3389/fmed.2021.695904.

5. de Parisot A, Kodjikian L, Errera $\mathrm{MH}$, et al. Randomized controlled trial evaluating a standardized strategy for uveitis etiologic diagnosis (ULISSE). Am J Ophthalmol. 2017;178:176-85. https://doi.org/10. 1016/j.ajo.2017.03.029.

6. Harris PA, Taylor R, Thielke R, Payne J, Gonzalez N, Conde JG. Research electronic data capture (REDCap) - a metadata-driven methodology and workflow process for providing translational research informatics support. J Biomed Inform. 2009;42(2): 377-81. https://doi.org/10.1016/j.jbi.2008.08.010.

7. Regulation (EU) 2016/679 of the European Parliament and of the Council of 27 April 2016 on the protection of natural persons with regard to the processing of personal data and on the free movement of such data, and repealing Directive 95/46/ EC (General Data Protection Regulation) (Text with EEA relevance). https://eur-lex.europa.eu/eli/reg/ 2016/679/oj. Accessed 3 Nov 2021.

8. Jabs DA, Nussenblatt RB, Rosenbaum JT, Standardization of Uveitis Nomenclature (SUN) Working Group. Standardization of uveitis nomenclature for reporting clinical data. Results of the First International Workshop. Am J Ophthalmol. 2005;140(3): 509-16. https://doi.org/10.1016/j.ajo.2005.03.057.

9. Standardization of Uveitis Nomenclature (SUN) Working Group. Development of classification criteria for the uveitides. Am J Ophthalmol. 2021;228: 96-105. https://doi.org/10.1016/j.ajo.2021.03.061.

10. Nussenblatt RB, Palestine AG, Chan CC, Roberge F. Standardization of vitreal inflammatory activity in intermediate and posterior uveitis. Ophthalmology. 1985;92(4):467-71. https://doi.org/10.1016/s01616420(85)34001-0.

11. Sen HN, Sangave AA, Goldstein DA, et al. A standardized grading system for scleritis. Ophthalmology. 2011;118(4):768-71. https://doi.org/10.1016/j. ophtha.2010.08.027.

12. McCluskey P, Wakefield D. Prediction of response to treatment in patients with scleritis using a standardised scoring system. Aust NZJ Ophthalmol. 1991;19(3):211-5. https://doi.org/10.1111/j.14429071.1991.tb00663.x.

13. Tugal-Tutkun I, Herbort CP, Khairallah M, Angiography Scoring for Uveitis Working Group (ASUWOG). Scoring of dual fluorescein and ICG inflammatory angiographic signs for the grading of posterior segment inflammation (dual fluorescein and ICG angiographic scoring system for uveitis). 
Int Ophthalmol. 2010;30(5):539-52. https://doi. org/10.1007/s10792-008-9263-X.

14. Chylack LT Jr, Wolfe JK, Singer DM, et al. The Lens Opacities Classification System III. The Longitudinal Study of Cataract Study Group. Arch Ophthalmol. 1993;111(6):831-6. https://doi.org/10.1001/ archopht.1993.01090060119035.
15. Kempf L, Goldsmith JC, Temple R. Challenges of developing and conducting clinical trials in rare disorders. Am J Med Genet A. 2018;176(4):773-83. https://doi.org/10.1002/ajmg.a.38413.MID: 28815894. 